\title{
Laparoscopic management of giant ovarian cysts in adolescents
}

\author{
Maciej Murawski, Andrzej Gołębiewski, Mariusz Sroka, Piotr Czauderna \\ Department of Surgery and Urology for Children and Adolescents, Medical University, Gdansk, Poland
}

Videosurgery and Other Miniinvasive Techniques 2012; 7 (2): 111-113

DOI: $10.5114 /$ wiitm.2010.22530

\begin{abstract}
A laparoscopic approach to giant ovarian cysts in girls, when the cyst's size exceeds the umbilicus, may be difficult regarding the risk of cyst rupture and limited working space. We present a series of three 12-year-old girls with giant ovarian cysts treated by percutaneous drainage under ultrasonographic guidance followed by laparoscopic resection. Clinical, laboratory and radiological diagnosis showed no signs of malignancy. After induction of general endotracheal anaesthesia, under ultrasonographic guidance, a $10 \mathrm{~F}$ vesicostomy catheter was placed into the cyst. Two to 4 l of serous (in one case mucinous) fluid were drained from the cyst. During laparoscopy, the abdominal cavity was inspected by the scope and no signs of malignancy were found. The laparoscopic procedure was completed with excision of the cyst and keeping some ovarian tissue in all cases. No intraoperative complications were observed. The mean operation time was $73 \mathrm{~min}$. Pathological examination revealed a mature cystic teratoma in two cases and mucinous cystadenoma in one. The postoperative recovery was uneventful in all girls and they were discharged home on postoperative day 2-4 with minimal pain. The ultrasound scans and tumour markers were normal on follow-up after 3 and 6 months. Laparoscopic excision of giant ovarian cysts after ultrasound-guided drainage seems to be a safe and applicable treatment modality in pre-menarchal girls with a normal tumour marker profile and benign features of the cyst, excluding the possibility of malignancy.
\end{abstract}

Key words: laparoscopy, ovary, cyst, minimally invasive surgery, children.

\section{Introduction}

Giant ovarian cysts in adolescents are very rare clinical entities [1, 2]. They are usually benign. The most commonly seen in children are functional cysts [1]. Nowadays the popularity of minimally invasive techniques is increasing and their advantages are obvious. However, a laparoscopic approach to giant ovarian cysts may be difficult because of the risk of cyst rupture and limited working space within the abdomen $[3,4]$.

We present a technique of percutaneous drainage of the cyst followed by its immediate laparoscopic removal. This method was used successfully in 3 consecutive patients.

\section{Case report}

Three 12-year-old girls were admitted to our department in the years 2006-2007 due to a huge abdominal cystic mass extending from the symphysis pubis to the epigastric region (Figure 1). Computed tomography (CT) scan of the abdomen and pelvis revealed a smooth surface cyst without septations, which corresponded to the diagnosis of benign ovarian cyst (Figure 2). Average maximum diameter of the cyst was $22 \mathrm{~cm}$ (range: 18-27 cm). The laboratory work-up was normal, including the oncological markers $\beta$-HCG, AFP, and CA-125. Clinical investigation and radiological tests excluded any signs of malignancy. 


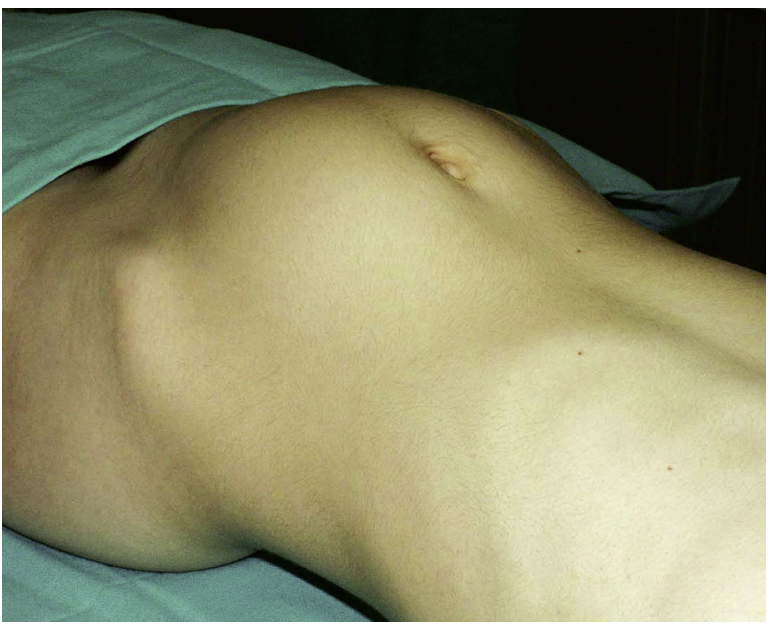

Figure 1. Patient on operating room table

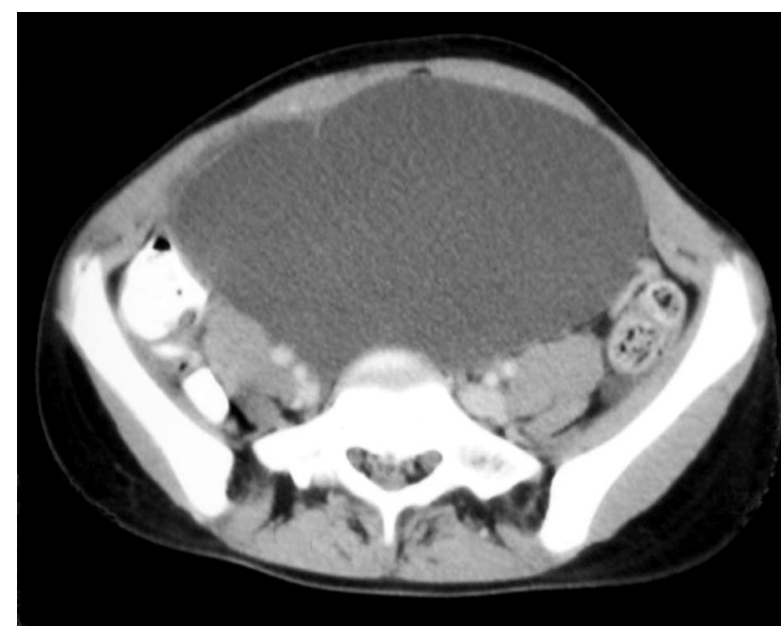

Figure 2. Computed tomography scan showing giant cystic lesion filling the entire abdominal cavity

Under general anaesthesia, with ultrasonographic guidance, a $10 \mathrm{~F}$ vesicostomy catheter was inserted into the cyst. Two to 4 I of serous (in one case mucinous) fluid were aspirated. Afterwards, a 10-mm trocar (camera port) was placed through the umbilicus by the open Hasson technique. Two additional trocars were inserted percutaneously in the right and left hypogastrium under direct vision. On laparoscopic examination a cyst originating from the left ovary in 1 case and the right ovary in 2 cases was seen. The cyst was excised, placed into the bag and removed through the umbilical incision. In all cases some ovarian tissue and ovarian tube was preserved. In all patients the contralateral ovary and uterus were normal.
The peritoneal cavity was lavaged with normal saline.

The mean operative time was 73 min (range: 60-90 min). The postoperative course was uneventful in all cases. Patients were discharged home on postoperative day 2, 4 and 3, respectively. Pathology revealed mature cystic teratoma in 2 cases and mucinous cystadenoma in 1 . Follow-up ultrasound scans and tumour markers were normal at 3 and 6 months follow-up.

\section{Discussion}

The largest ovarian tumour ever weighed $137.4 \mathrm{~kg}$ and was removed intact in 1994 by O'Hanlan [5]. In paediatric surgery we usually deal with much smaller abdominal masses. Ovarian cysts are labelled as large when they are over $5 \mathrm{~cm}$ and giant when they are over $15 \mathrm{~cm}$ [4]. In children, however, it is better to compare the size of the cyst to the size of the peritoneal cavity. Some authors have defined giant ovarian cysts as those reaching above the level of the umbilicus [6]. We strongly agree with this statement.

Giant ovarian cysts in adolescents, especially in pre-menarchal girls, are very rare $[1,2]$. The estimated incidence of ovarian tumours (benign and malignant) is 2.6 cases per 100000 in girls younger than 15 years of age [2]. Of these, about 35\% are benign [2]. Cysts that reach such a giant size are almost always benign, but careful preoperative diagnosis (imaging and tumour markers) should be carried out due to the suspicion of malignancy [4]. When the malignancy risk is high, the laparoscopic approach is inappropriate.

Giant ovarian cysts always require resection, because of associated symptoms due to mass effect, difficulties in establishing the origin of the mass and the above-mentioned risk of malignancy [4].

Presently laparoscopy is widely used in paediatric surgery. The advantages of minimally invasive techniques are well known, and include better cosmesis, less pain and shorter hospital stay. Additionally, laparoscopy can easily determine the origin of the cyst [4].

However, decompression of the mass is required to remove it laparoscopically. Possible options include: 1) ultrasound-guided drainage of the cyst, 2) decompression of the mass via minilaparotomy, 3) laparoscopic-guided aspiration [1, 3, 4]. On the other hand, decompression allows one to avoid cyst per- 
foration during trocar insertion during laparoscopy. Leakage of the cyst contents may result in chemical peritonitis or dissemination of cells in the case of malignant tumour [1, 3]. Although the significance of this leakage is controversial, we suggest avoiding it whenever possible $[3,5,7]$. This can be achieved in our opinion by percutaneous drainage of the cyst and insertion of the first trocar according to the Hasson technique [8].

\section{Conclusions}

Laparoscopic excision of a giant ovarian cyst after percutaneous ultrasound-guided drainage seems to be safe and effective. We believe that with careful preoperative assessment and adequate attention to avoiding spillage during surgery, the benefits of laparoscopic access can be offered to young girls irrespective of the size of the ovarian cyst.

\section{References}

1. Ates O, Karakaya E, Hakguder G, et al. Laparoscopic excision of a giant ovarian cyst after ultrasound-guided drainage. J Ped Surg 2006; 41: E9-11.

2. Sri Paran T, Mortell A, Devaney D, et al. Mucinous cystoadenoma of the ovary in perimenarchal girls. Pediatr Surg Int 2007; 22: 224-7.

3. Goh SM, Yam J, Loh F, et al. Minimal access approach to the management of large ovarian cysts. Surg Endosc 2007; 21: 80-3.

4. Dolan MS, Boulanger SC, Salameh JR. Laparoscopic management of giant ovarian cyst. JSLS 2006; 10: 254-6.

5. Einenkel J, Alexandre H, Schotte D. Giant ovarian cysts: Is a pre- and intraoperative drainage an advisable procedure? Int J Gynecol Cancer 2006; 16: 2039-43.

6. Eltabbakh GH, Charboneau AM, Eltabbakh NG. Laparoscopic surgery for large benign ovarian cysts. Gynecologic Oncology 2008; 108: 72-6.

7. Rabbani I, Wynn JS, Hickling DJ. Laparoscopic excision of a large ovarian cyst. Gynecol Surg 2007; 4: 225-7.

8. Sagiv R, Golan A, Glezerman M. Laparoscopic management of extremely large ovarian cysts. Obstet Gynecol 2005; 105: 1319-22. 